\title{
Guidelines on hypertension in 2005: a commentary
}

\author{
Edward D Frohlich
}

The increasing availability of new fundamental information on physiologic, pharmacologic and other biologic factors, as well as on demographic, epidemiologic and clinical features of hypertension and associated cardiovascular and renal disease has dramatically advanced our concept and treatment of hypertension. Innovations arise faster than the guidelines can keep pace, and for practice to stay up to date some important features must be appreciated.

First, in the US, only $34 \%$ of hypertensive individuals are aware of their disease and have it controlled with appropriate therapy. Although shocking, these data are better than in other nations.

Second, the term prehypertension has been introduced, with the hope that development of sustained hypertension can be prevented with lifestyle modifications in adults who have blood pressures of $120-139 / 80-89 \mathrm{mmHg}$. Although well intentioned, the labeling of patients in this way has created some disadvantageous consequences that might impact on adherence to the guidelines, such as raised insurance premiums or problems for people wishing to donate kidneys.

A third issue concerns the recommendation to prescribe a thiazide diuretic as initial antihypertensive therapy for all patients with uncomplicated hypertension. Although it has been perceived as mandatory, this is only a guideline; use of other drug classes is permitted in more-complicated circumstances. Further confusion relates to the recommendation to initiate antihypertensive therapy with multiple agents for stage 2 hypertension $(>160 />100 \mathrm{mmHg})$. Although the rationale might seem reasonable, use of multiple agents can lead to adverse effects and frequently might be unnecessary. If supervision is required, office visits seem reasonable before increasing the number of drugs. Thus, broadening the range of blood pressures in stage 2 hypertension does not seem (I believe) valid.
Innovations

arise faster

than the

guidelines can

keep pace, and

for practice

to stay up to

date some

important

features

must be

appreciated.

ED Frohlich is the

Alton Ochsner

Distinguished

Scientist, Ochsner

Clinic Foundation,

New Orleans, LA,

USA.

Competing interests

The author declared he has

no competing interests.

www.nature.com/clinicalpractice doi:10.1038/ncpcardio0223
Finally, a concern with the seventh Joint National Committee's report is that cardiac failure is considered as one topic. Although hypertension remains the major cause of cardiac failure (and hospitalization of patients older than 65 years), it no longer mainly results from impaired systolic ventricular function because of inadequate blood pressure control. Most hypertension now relates primarily to impaired diastolic function in the presence of preserved systolic function. Treatment strategies for these two forms of cardiac failure can differ substantially and, therefore, should be given broader consideration.

Given these issues, what therapeutic goals should we be aiming to achieve in 2005? For most hypertensive patients the ideal target blood pressure is $<140 /<90 \mathrm{mmHg}$. Several multicenter trials involving patients with diabetes mellitus or chronic renal disease, however, suggest setting a lower target such as $<130 /<80 \mathrm{mmHg}$. To me, the findings also suggest that this goal should also be recommended for patients with the metabolic syndrome. If the hemoglobin $\mathrm{A}_{1 \mathrm{C}}$ level is $>6.0-6.5 \%$, moreaggressive therapy can be used to reduce pressure and preserve renal function. At least two drugs, of which one should be a diuretic, might have to be used routinely. In patients with impaired renal function, a loop-acting diuretic (e.g. furosemide) might be necessary; the dose can be increased incrementally until the desired response is achieved.

The cardiovascular risk associated with elevated systolic or diastolic pressure is linear, and lower goal pressures might mean improved cardiovascular, renal and cerebral outcomes. A strict perception of goal targets is particularly important for high-risk groups, but, most importantly, all health-care providers must take particular care to detect patients with elevated blood pressures, to evaluate them carefully and to bring pressures to within prescribed levels. 